\title{
Rapid and sensitive detection of Bordetella bronchiseptica by loop-mediated isothermal amplification (LAMP) ${ }^{1}$
}

\author{
Hui Zhang ${ }^{2}$, Ling Zhu², Yuancheng Zhou'², Hongwei $\mathrm{Ji}^{2}$, Hongbo Dai ${ }^{2}$, Wanzhu Guo ${ }^{2}$ \\ and Zhiwen $\mathrm{Xu}^{2 *}$
}

\begin{abstract}
Zhang H., Zhu L., Zhou Y.C., Ji H.W., Dai H.B., Guo W.Z. \& Xu Z.W. 2013. Rapid and sensitive detection of Bordetella bronchiseptica by loop-mediated isothermal amplification (LAMP). Pesquisa Veterinária Brasileira 33(10):1222-1226. Key Laboratory of Animal Biotechnology Center of Sichuan Province, College of Veterinary Medicine of Sichuan Agricultural University, Ya'an, Sichuan, 625014, PR China. E-mail:_abtcxzw@126.com

Bordetella bronchiseptica causes acute and chronic respiratory infections in diverse animal species and occasionally in humans. In this study, we described the establishment of a simple, sensitive and cost-efficient loop-mediated isothermal amplification (LAMP) assay for the detection of $B$. bronchiseptica. A set of primers towards a $235 \mathrm{bp}$ region within the flagellum gene of $B$. bronchiseptica was designed with online software.. The specificity of the LAMP assay was examined by using 6 porcine pathogens and 100 nasal swabs collected from healthy pigs and suspect infected pigs. The results indicated that positive reactions were confirmed for all B. bronchiseptica and no cross-reactivity was observed from other non- $B$. bronchiseptica. In sensitivity evaluations, the technique successfully detected a serial dilutions of extracted $B$. bronchiseptica DNA with a detection limit of 9 copies, which was 10 times more sensitive than that of PCR. Compared with conventional PCR, the higher sensitivity of LAMP method and no need for the complex instrumentation make this LAMP assay a promising alternative for the diagnosis of $B$. bronchiseptica in rural areas and developing countries where there lacks of complex laboratory services.
\end{abstract}

INDEX TERMS: LAMP, Bordetella bronchiseptica, flagellum gene, detection, sensitivity.

\section{INTRODUCTION}

Bordetella bronchiseptica (B. bronchiseptica) is thought to be an evolutionary progenitor of Bordetella pertussis and Bordetella parapertussis, which are causative agents of whooping cough in humans (Mattoo \& Cherry 2005). It is a pathogenic bacterium with a broad range of hosts and causes chronic infections: kennel cough in dogs, snuffles in rabbits, and atrophic rhinitis in swine (Goodnow 1980). Human infections occasionally occur, most frequently in immunocompromised individuals, but related illness in healthy adults and children has also been reported (Stojanov et al. 2000, Senzilet et al. 2001, Strebel et al. 2001). Research findings suggest that the concurrent presence of B. bron-

\footnotetext{
${ }^{1}$ Received on April 18, 2013.

Accepted for publication on September 12, 2013.

2 Key Laboratory of Animal Biotechnology Center of Sichuan Province, College of Veterinary Medicine of Sichuan Agricultural University, Ya'an, Sichuan, 625014, PR China. *Corresponding author: abtcxzw@126.com
}

chiseptica and other respiratory pathogens results in more severe disease than infection with $B$. bronchiseptica alone (Brockmeier et al. 2000, Brockmeier 2004, Brockmeier et al. 2008). In pigs, B. bronchiseptica is a primary etiologic agent of swine atrophic rhinitis and primary bronchopneumonia in piglets (Underdahl et al. 1982), the most important of it is increasing incidence and severity of respiratory system disease through cooperate with other pathogenic germs (Dugal et al. 1992), for example, B. bronchiseptica and Pasteurella multocida are etiologic agents of progressive atrophic rhinitis (PAR) in swine. The outbreak of PAR causes serious economic losses because they are often accompanied by a reduced growth rate and inefficient feed conversion (Chanter et al. 1989). Therefore, it is great of importance to diagnose the germ in the early stages of surveillance of the disease and for control of bacterial spread. To the best of our knowledge, the detection of B.bronchiseptica infections in a laboratory is traditionally based primarily on culture, though highly specific, takes a long time to isolate and lacks sufficient sensitivity ÿfurthermore, visual recognition of 
suspect $B$. bronchiseptica colonies requires a certain level of skill and experience. For rapid and sensitive diagnosis, polymerase chain reaction (PCR), as says, which revolutionized the laboratory diagnosis of $B$. bronchiseptica infections (Hozbor et al. 1999), have previously been found to be at most a useful complement to culture, with regard to both sensitivity and rapidity of the test. However, despite its excellent sensitivity and specificity, complicated procedures and the expensive systems required makes it difficult for widespread use. Clearly, a simpler, more rapid, and more sensitive diagnostic assay is needed.

LAMP is a novel nucleic acid amplification method that could amplify target genes under isothermal conditions with the advantage of being rapid, simple and easy to perform as reported by Notomi and his colleagues(Notomi et al., 2000). The principle of LAMP is based on autocycling strand displacement DNA synthesis in the presence of exonuclease-negative Bst DNA polymerase, with four specifically designed primers that recognize six distinct regions of the target DNA, especially in 2002, Nagamine et al. reported that add two additional loop primers could reduce the reaction time and has the potential to increase sensitivity lead to this technology has a great development (Nagamine et al. 2002). It has higher specificity and amplification efficiency compared to PCR as a gene amplification technology, so in recent years, this technology has been widely used for the detection of various pathogenic microorganisms, such as Actinobacillus pleuropneumoniae (Yang et al. 2009), the

Table 1. Primers of flagellum gene based LAMP and PCR for Bordetella bronchiseptica detection

\begin{tabular}{|c|c|c|c|}
\hline $\begin{array}{l}\text { Primer } \\
\text { name }\end{array}$ & Type & Length & Sequence $\left(5^{\prime}-3^{\prime}\right)$ \\
\hline$F 3^{a, b}$ & $\begin{array}{l}\text { Forward outer } \\
\text { primer }\end{array}$ & 18 & CCTTTGCCGCCAGATTCC \\
\hline$B 3^{a, b}$ & $\begin{array}{c}\text { Backward outer } \\
\text { primer }\end{array}$ & 19 & GCTCCCAAGAGAGAAAGGC \\
\hline FIP $^{a}$ & $\begin{array}{l}\text { Forward inner } \\
\text { primer(F1c-F2) }\end{array}$ & 43 & $\begin{array}{l}\text { TGGATTACGGCAGGTTTGCGA- } \\
\text { TTTT-CCCGCACATTTCCGAACT }\end{array}$ \\
\hline $\mathrm{BIP}^{\mathrm{a}}$ & $\begin{array}{l}\text { Backward inner } \\
\text { primer (B1-B2c) }\end{array}$ & 43 & $\begin{array}{l}\text { GGCAACAAAGGAAATCGCGGC- } \\
\text { TTTT-TCTTCAAACCGGGCAGCT }\end{array}$ \\
\hline Loop $F^{a}$ & $\begin{array}{l}\text { Forward loop } \\
\text { primer }\end{array}$ & 17 & ACTTAAGCAAAAAAAGT \\
\hline Loop $B^{a}$ & $\begin{array}{l}\text { Backward loop } \\
\text { primer }\end{array}$ & 15 & TTACAGATGGGCGGC \\
\hline
\end{tabular}

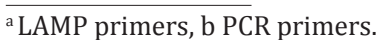

avian influenza virus (Dinh et al. 2011), and the lassa virus (Fukuma et al. 2011). Based on our work and that of others, we propose a LAMP assay for the detection of B. bronchiseptica targeting the flagellum gene.

\section{MATERIALS AND METHODS}

\section{Bacterial strains and DNAs preparation}

Bordetella bronchiseptica was used as positive control for the development and optimization of the LAMP assays. To determine the specificity of LAMP assayÿthe following pathogens were used in present study: B. pertussis, Haemophilus parasuis, Pasteurella multocida, Streptococcus suis, Escherichia coli, A. pleuropneumoniae. Some bacterial strains used in this study were obtained from China Institute of Veterinary Drug Control (CIVDC). B. bronchiseptica were grown on Bordet-Gengou (BG) agar (BD Biosciences, San Jose, CA) supplemented with 7.5\% defribinated sheep blood (Hardy Diagnostics, Santa Maria, CA) or Stainer-Scholte (SS) broth at $37^{\circ} \mathrm{C}$. A pleuropneumoniae were grown overnight in tryptic soy broth (TSB) medium at $37^{\circ} \mathrm{C}$, supplemented with $10 \mu \mathrm{g}$ of $\mathrm{NAD} / \mathrm{ml}$ and $10 \%$ bovine serum. Other strains were cultured in Luria broth (LB) supplemented $10 \%$ bovine serum at $37^{\circ} \mathrm{C}$ for $18 \mathrm{~h}$. Bacterial genomic DNAs were extracted by using a QIAamp DNA mini kit (QIAGEN Inc; Valencia, CA, USA) according to the manufacturer's instructions.

\section{LAMP primer design}

The conserved $B$. bronchiseptica flagellum gene sequence (Genbank accession number EU327790, AJ012319, L13034) was selected and used as the target for LAMP primers design. Their sequence homologies were determined using DNAStar software and the conserved regions with the highest levels of homology were chosen as templates for LAMP primers design using primer Explore V4 (http://primerexplorer.jp/elamp4.0.0 /index.html). The specificity of the designed primers was confirmed by BLAST on the National Center for Biotechnology Information (NCBI) server (http://www.ncbi.nlm.nih.gov/). The name and sequence of each primer are shown in Table 1, and the location of oligonucleotide primers are shown in Fig.1. All the primers were synthesized at Shanghai Sangon Biological Engineering Technology and Service Co., Ltd. (Shanghai, China).

\section{Optimization of LAMP reaction conditions}

The LAMP reaction was carried out with $25 \mu \mathrm{L}$ of a mixture containing $1.0 \mu \mathrm{L}$ of each of the F3 and B3 primers $(5 \mu \mathrm{M}), 1.0 \mu \mathrm{L}$ of each of the BIP and FIP primers $(40 \mu \mathrm{M}), 1.0 \mu \mathrm{L}$ of each of the Loop F and Loop B primers $(20 \mu \mathrm{M}), 8 \mathrm{U}$ of Bst DNA polymerase (8U/ $\mu \mathrm{L}$, New England Biolabs), $2.5 \mu \mathrm{L} 10 \times B s t$ DNA polymerase reaction buffer (containing 200mM Tris- $\mathrm{HCl}$ (pH 8.8), $100 \mathrm{mM} \mathrm{KCl,} 100$ $\mathrm{mM}\left(\mathrm{NH}_{4}\right)_{2} \mathrm{SO}_{4}, 20 \mathrm{mM} \mathrm{MgSO}_{4 \ddot{\mathrm{y}}} 1 \%$ Triton X-100), $5.5 \mu \mathrm{L}$ of Betaine (5M), 3.0 $\mu \mathrm{L}$ of $\mathrm{MgCl}_{2}(30-70 \mathrm{mM}), 5.0 \mu \mathrm{L}$ of dNTP $\operatorname{Mix}(10 \mathrm{mM})$, and

\section{TGGCGCCTGCCCTATCCCGTCCGCGCCGCACGGACGCCTGTCCCCGCAGGAACATGC $\overline{\mathrm{CCT}}$} F3 F2 Loop F

$121 \overline{\text { TTGCCGCCAGATTCC }} \overline{\text { CCCGCACATTTCCGAACT }}$ TC $\overline{\text { ACTTTTTTTGCTTAAGT }}$ CCG $\overline{\overline{\text { TCGCA }}}$ F1C B1C

181 AACCTGCCGTAATCCA GGCAACAAAGGAAATCGCGGCGCTGTGCAAGCGAAAGTCCGATG Loop B

B2 B3

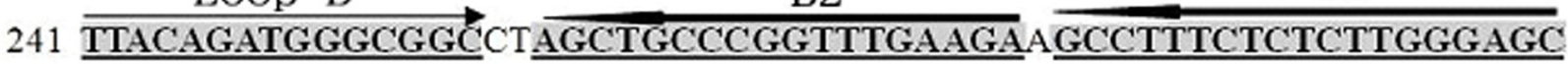
Fig.1. Location of oligonucleotide primers used for LAMP assay of Bordetella bronchiseptica in partial flagellum gene. 
2.0 $\mu \mathrm{L}$ DNA template(containing $1 \times 10^{2}$ copies per microtube, the standard DNA copy number was calculated using the equations described by Luo et al(Luo et al. 2012)). The mixture was incubated at $\left(63-67^{\circ}\right)$ for $60 \mathrm{~min}$ and then heated at $80^{\circ}$ for $5 \mathrm{~min}$ in a water bath to terminate the reaction. In a previous report Kamachi et al. 2006, the LAMP with heat-denatured template DNA was 100 times more sensitive than that with no-denatured template, therefore, before the reaction, the reaction mixture (without the Bst DNA polymerase) was heated at $96^{\circ} \mathrm{C}$ for $5 \mathrm{~min}$ and immediately cooled on ice for $3 \mathrm{~min}$. Finally, the LAMP products were evaluated by a $2.0 \%$ agarose gel electrophoresis (visualized within 60 minutes with UV light at $365 \mathrm{~nm}$ as recommended by the manufacturer).

To confirm the amplified sequence, we digested the LAMP product and submitted to TakaRa (Dalian, China) for sequencing.

\section{PCR assays for Bordetella bronchiseptica detection}

To evaluate the sensitivity of LAMP reaction, conventional PCR was performed using LAMP outer primers (F3 and B3). The reaction mixture contained $2 \mu \mathrm{L}$ DNA template, $1 \mu \mathrm{L}$ of each primer (each $5 \mu \mathrm{M}$ ), $10 \mu \mathrm{L} 2 \times$ long Tag PCR Master Mix and distilled water was added to a total volume of $20 \mu \mathrm{L}$. PCR amplification was achieved by initial denaturation at $95^{\circ} \mathrm{C}$ for $5 \mathrm{~min}$ followed by 40 cycles each included denaturation step at 95 ! for 30 s, annealing step at $56^{\circ} \mathrm{C}$ for 30 s and extension step at 72 ! for 30 s, and then a final extension step at $72^{\circ} \mathrm{C}$ for $10 \mathrm{~min}$ was included. The reaction products were kept at $4^{\circ} \mathrm{C}$ until analyzed by $1.2 \%$ agarose gel electrophoresis(visualized within 60 minutes with UV light at 365 $\mathrm{nm}$ as recommended by the manufacturer).

\section{Specificity and sensitivity of LAMP method}

To determine the specificity of the primers for LAMP amplification, 6 potential cross-reactions bacteria (see section 2.1) that are likely to be associated with the $B$. bronchiseptica were tested. The extracted B.bronchiseptica DNA was used as the positive control, and DNAs extracted from other bacterial were used as negative control. Reaction conditions were carried out as described above. In order to evaluate the sensitivity of LAMP assay for detecting B.bronchiseptica, serial dilutions of the extracted DNA of B. bronchiseptica containing $9 \times 10^{6}, 9 \times 10^{5}, 9 \times 10^{4}, 9 \times 10^{3}, 9 \times 10^{2}$, $9 \times 10^{1}, 9 \times 10^{0}$ and $9 \times 10^{-1}$ copies per a $0.2 \mathrm{ml}$ microtube were prepared (measured using a NanoDrop 2000 spectrophotometer), the copy number was calculated using the following formula (Luo et al. 2012):

Number copiesÿtube $=$

$=6.02 \times 10^{23}($ copiesÿmol $) \times$ concentration $(g \ddot{y} \mu \mathrm{L}) \times 2 \mu \mathrm{L}$ tube MW $(\mathrm{g}$ ÿmol $)$

PCR assay was carried out using the same dilution as described above for the LAMP assay. Sensitivity tests were repeated in triplicate and the lowest limits of detection were reported.

\footnotetext{
Application of the LAMP assay in clinical and experimental samples

The usefulness of the LAMP assay was further evaluated by using 50 nasopharyngeal swabs $(n=50)$ obtained from healthy pigs, 50 nasopharyngeal swabs (from different pig herds) $(n=50)$ suspect to be infected with atrophic rhinitis in Sichuan province. All the Bacterial genomic DNAs were extracted by using a QIAamp DNA mini kit (QIAGEN Inc; Valencia, CA, USA) according to the manufacturer's instructions. All the specimens were tested by culture, conventional PCR and LAMP assays in parallel. The test results from the three methods were compared with each other and calculations of positive detection rates were performed.
}

\section{RESULTS}

\section{Optimum LAMP reaction conditions for Bordetella bronchiseptica detection \\ Specificity of the LAMP assay. The specificity of the} LAMP reaction system was confirmed by using 6 species of bacteria that are likely to be associated with the Bordetella bronchiseptica. Agarose gel electrophoresis (Fig.2) showed that positive results showed a ladder pattern with a set of bands of different sizes appeared only using the DNA from the $B$. bronchiseptica as template, in contrast, no LAMP products were detected in the reactions that performed with DNA harvested from the 6 other Bacteria. Additionally, the amplified products, which resulted in an observable white turbidity in the reaction tube due to formation of magnesium pyrophosphate from positive LAMP reaction, could be directly inspected by the naked eye after centrifuged at $12000 \mathrm{rpm}$ for $1 \mathrm{~min}$. These results indicated that this method could be used to specifically detect $B$. bronchiseptica without cross-reactivity with any other Bacterial present in this study.

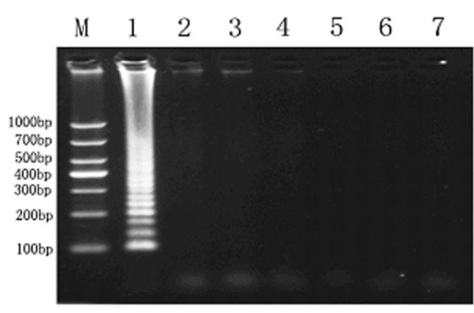

(A)

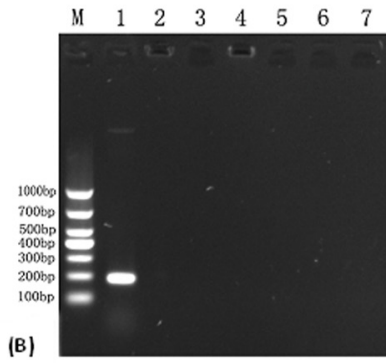

(B)
Fig.2. Specificity of LAMP (A) and PCR (B) assay tested by electrophoretic for B.bronchiseptica detection. Lane M, DNA size Marker (DL 1000); Lane1, Bordetella bronchiseptica; Lane29, B. pertussis, Haemophilus parasuis, Pasteurella multocida, Streptococcus suis, Escherichia coli, and Actinobacillus pleuropneumoniae, respectively.

Sensitivity of the LAMP assay. To determine the minimum number of genome sequences detectable by the LAMP method, 10-fold serial dilutions of template DNA extracted from $B$. bronchiseptica were usedÿand compared this with PCR by using the same templates at identical concentrations. As observed on $2.0 \%$ agarose gel electrophoresisthe results in Figure 3 showed that the detection limit of LAMP assay being estimated to be 9 copies/tube. PCR amplified a 177-bp DNA product that reached the sensitivity limit of $9 \times 10^{1}$ copies/tube. These results suggested that the LAMP assay in this study was approximately 10 times more sensitive than that of PCR assay. The increased sensitivity of LAMP assay for detecting B. bronchiseptica over the PCR assay indicates that the LAMP assay developed in the current study is suitable for B. bronchiseptica diagnosis.

Evaluation of LAMP assays on clinical isolates. In order to assess the applicability of the LAMP assay for detecting B. bronchiseptica in clinical material, 50 nasopharyngeal swabs obtained from healthy pigs and 50 nasopharyngeal swabs from suspected to be infected with atrophic rhinitis were tested by LAMP assay and simultaneously by culture 


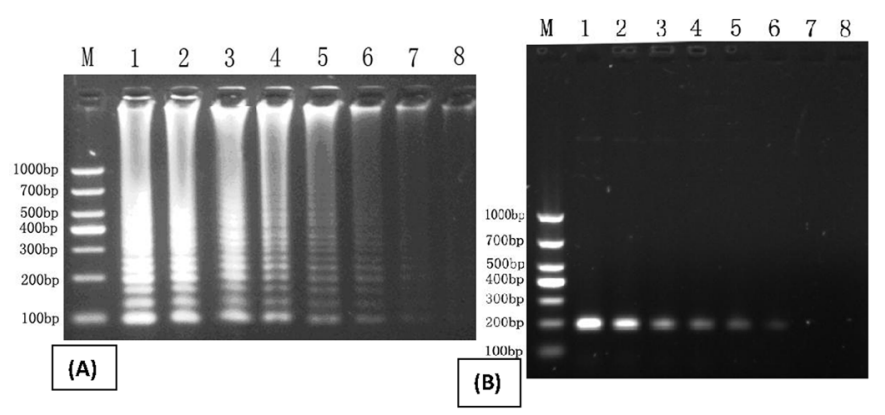

Fig.3. Sensitivities of LAMP and PCR assays for detection of Bordetella bronchiseptica. (A) Agarose gel analysis of the LAMP sensitivity, (B) agarose gel analysis of the PCR sensitivity. Lane M, DNA size Marker (DL 1000); Lane1-8, reaction conducted using 10 -fold serial dilutions of $B$. bronchiseptica DNA from $9 \times 10^{6}$ to $9 \times 10^{-1}$ copies/tube.

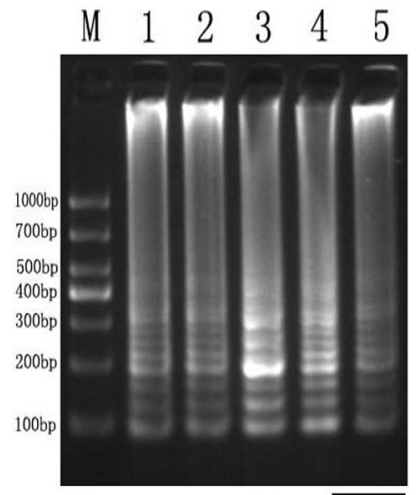

(A)

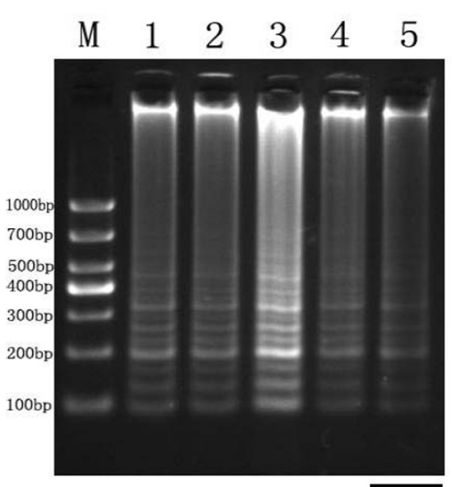

(B)
Fig.4. Optimization of the LAMP reaction for Bordetella bronchiseptica detection. (A) Effect of $\mathrm{Mg}^{2+}$ on the LAMP reaction: Lane M, DNA size Marker (DL 1000). Lanes 1-5, $\mathrm{Mg}^{2+}$ concentration was $30,40,50,60$, and $70 \mathrm{mM}$, respectively. (B) The effect of temperature on the LAMP reaction: Lane M, DNA size Marker (DL 1000). Lanes 1-5, temperatures of 63, 64, 65, 66 and 67 !, respectively.

Table 2. Comparative analysis of Bordetella bronchiseptica detection by LAMP, PCR and culture

\begin{tabular}{lccccc}
\hline Assays & \multicolumn{3}{c}{ Number of positive samples } & & \multicolumn{3}{c}{ Percentage of positive samples (\%) } \\
\cline { 2 - 3 } \cline { 5 - 6 } $\begin{array}{l}\text { Healthy } \\
\text { material }\end{array}$ & $\begin{array}{c}\text { Suspected to } \\
\text { be infected }\end{array}$ & & $\begin{array}{c}\text { Healthy } \\
\text { material }\end{array}$ & $\begin{array}{c}\text { Suspected to } \\
\text { be infected }\end{array}$ \\
\hline LAMP & 0 & 27 & $0 \%$ & $54 \%$ \\
PCR & 0 & 20 & $0 \%$ & $40 \%$ \\
Culture & 0 & 15 & $0 \%$ & $30 \%$
\end{tabular}

methods as well as conventional PCR. The results of three methods are summarized in Table 2. Briefly, among the 50 samples collected from healthy pigs, there was no false positivity for the three assays. For the 50 suspicious samples, the LAMP Method gave a total of 27 positive results and 7 samples were positive for LAMP but negative for the PCR method. 15 out of the 50 samples were isolated by plating, moreover, all samples positive by isolating were also positive for LAMP and PCR assay. These results indicated that LAMP assay exhibited better sensitivities than PCR and cultivation when detecting B.bronchiseptica in the field samples.
Confirmation of LAMP product. In order to rule out the possibility of false positivity, all the positive products of LAMP were purified by gel purification kit (Omega) according to the manufacture's protocol, then submitted to the TakaRa (Dalian, China) for sequencing and the results were as expected.

\section{DISCUSSION}

Bordetella bronchisepticais involved in the aetiology of atrophic rhinitis and bronchopneumonia, which increase incidence and severity of respiratory system disease through cooperate with other pathogenic germs, so B. bronchiseptica is a contributory agent in the porcine respiratory disease complex, a multifactorial diseases that is increasingly problematic for swine producers (Brockmeier et al. 2002). Traditionally, to identify B.bronchiseptic infection, definitive diagnosis of infection with $B$. bronchiseptica is established from clinical signs and isolation from nasal swabs or lungs, and suspect colonies are used to biochemical testing, however, it is a fact that $B$. bronchiseptica are often found in low numbers compared to the other bacteria because of it may be masked by other bacteria which growing faster than B. bronchiseptica (Register et al. 1998). In the past decades, routine PCR has emerged as an important method used widely for the laboratory diagnosis because of its rapidity, sensitivity and specificity.

LAMP has successfully been used to diagnose pathogenic infections in humans and animals(Liu et al. 2009, Yang et al. 2009, Fukuma et al. 2011). Compared with other assaysÿthe LAMP method has several advantages. To begin with, it has high specificity and sensitivity. The amplification of LAMP products depend on the four specifically designed primers that recognize six distinct regions of the target DNA, so it has a high specificity than other assays, meanwhile, in this study, the detection of nine copies of $B$. bronchiseptica DNA per reaction which is 10 times more sensitive than that of PCR assay. In 1999, Hozbor et al (Hozbor et al. 1999) developed a PCR assay for B. bronchiseptica detection, this method made great contribution to the diagnosis of B. bronchiseptica. However, PCR requires special equipment, technical expertise, and 2-3h for amplification, making it difficult to adapt for rapid detect in the field. Second, LAMP analysis is rapid with a short reaction time that only need less than $1 \mathrm{~h}$ compared with a conventional PCR time of at least $2 \mathrm{~h}$. Third, inexpensive, its operational simplicity and isothermal nature make it ideally suited for use in the field. In PCR, thermal cycling is required to denature the template, anneal primers and extend the amplicon, conversely, the assay was performed at isothermal conditions using a conventional water bath or heat block. It does not rely on expensive instruments, reagents or post-amplification analysis.

In summary, we successfully developed a simple and rapid LAMP assay for the detection of $B$. bronchiseptica. Using the LAMP assays described in this study, the positive sample resulted in a characteristic ladder of multiple bands. In contrast, no visible bands were detected when the 6 other pig bacteria encountered frequently or healthy material were used for the analysis, shown its specificity for $B$. 
bronchiseptica detection. In sensitivity evaluations, LAMP was possible to detect 9 copies, but that derive for PCR was 90 copies. Thus, the LAMP method established in this study was 10 times more sensitive than the conventional PCR, which is in agreement with most previous reports (Liu et al. 2009, Yang et al. 2009, Fukuma et al. 2011). Of the 50 field samples in this study 7 samples were identified as B. bronchiseptica positive by LAMP while conventional PCR presented negative results, making LAMP the obvious choice for diagnosing $B$. bronchiseptica in clinical practice. Overall, the LAMP method developed in the present study demonstrated higher sensitivity than that of PCR and cultivation. Due to its simplicity and rapidityÿit is a promising assay for extensive application for the rapid detection of genetically modified ingredients in the laboratory and the field.

\section{CONCLUSIONS}

A novel system for the rapid and easy identification of Bordetella bronchiseptica was described which was rapid, highly sensitive, simple, and inexpensive.

This method can potentially be used for tests in the field.

Acknowledgement.- This study was funded by Projects in Sichuan Science and Technology Pillar Program: the key technology of modern pig industry chain research integration and industrialization demonstration (2012NZ0001).

\section{REFERENCES}

Brockmeier S.L. 2004. Prior infection with Bordetella bronchiseptica increases nasal colonization by Haemophilus parasuis in swine. Vet. Microbiol. 99:75-78.

Brockmeier S.L., Loving C.L., Nicholson T.L. \& Palmer M.V. 2008. Coinfection of pigs with porcine respiratory coronavirus and Bordetella bronchiseptica. Vet. Microbiol. 128: 36-47.

Brockmeier S.L., Palmer M.V. \& Bolin S.R. 2000. Effects of intranasal inoculation of porcine reproductive and respiratory syndrome virus, Bordetella bronchiseptica, or a combination of both organisms in pigs. Am. J. Vet. Res. 61:892-899.

Brockmeier S.L., Register K.B., Magyar T., Lax A.J., Pullinger G.D. \& Kunkle R.A. 2002. Role of the dermonecrotic toxin of Bordetella bronchisepti$c a$ in the pathogenesis of respiratory disease in swine. Infect. Immun. 70:481-490.

Chanter N., Magyar T. \& Rutter J.M. 1989. Interactions between Bordetella bronchiseptica and toxigenic Pasteurella multocida in atrophic rhinitis of pigs. Res. Vet. Sci. 47:48-53.

Dinh D.T., Le M.T., Vuong C.D., Hasebe F. \& Morita K. 2011. An updated loop-mediated isothermal amplification method for rapid diagnosis of H5N1 Avian Influenza Viruses. Trop. Med. Health. 39:3-7.
Dugal F., Belanger M. \& Jacques M. 1992. Enhanced adherence of Pasteurella multocida to porcine tracheal rings preinfected with Bordetella bronchiseptica. Can. J. Vet. Res. 56:260-264.

Fukuma A., Kurosaki Y., Morikawa Y., Grolla A., Feldmann H. \& Yasuda J. 2011. Rapid detection of Lassa virus by reverse transcription-loop-mediated isothermal amplification. Microbiol. Immunol. 55:44-50.

Goodnow R.A. 1980. Biology of Bordetella bronchiseptica. Microbiol. Rev. 44:722-738.

Hozbor D., Fouque F. \& Guiso N. 1999. Detection of Bordetella bronchisepti$c a$ by the polymerase chain reaction. Res. Microbiol. 150:333-341.

Kamachi K., Toyoizumi-Ajisaka H., Toda K., Soeung S.C., Sarath S., Nareth Y., Horiuchi Y., Kojima K., Takahashi M. \& Arakawa Y. 2006. Development and evaluation of a loop-mediated isothermal amplification method for rapid diagnosis of Bordetella pertussis infection. J. Clin. Microbiol. 44:1899-1902.

Liu M., Luo Y., Tao R., He R., Jiang K., Wang B. \& Wang L. 2009. Sensitive and rapid detection of genetic modified soybean (Roundup Ready) by loop-mediated isothermal amplification. Biosci. Biotechnol. Biochem. 73:2365-2369.

Luo J.G., Ge J.W., Tang L.J., Qiao X.Y., Jiang Y.P., Cui W., Liu M. \& Li Y.J. 2012. Development of a loop-mediated isothermal amplification assay for rapid detection of bovine parvovirus. J. Virol. Methods. 44:1899-1902.

Mattoo S. \& Cherry J.D. 2005. Molecular pathogenesis, epidemiology, and clinical manifestations of respiratory infections due to Bordetella pertussis and other Bordetella subspecies. Clin. Microbiol. Rev. 18: 326-382.

Nagamine K., Hase T. \& Notomi T. 2002. Accelerated reaction by loop-mediated isothermal amplification using loop primers. Mol. Cell. Probes $16: 223-229$

Notomi T., Okayama H., Masubuchi H., Yonekawa T., Watanabe K., Amino N. \& Hase T. 2000. Loop-mediated isothermal amplification of DNA. Nucleic. Acids. Res. 28:E63.

Register K.B., Lee R.M. \& Thomson C. 1998. Two-color hybridization assay for simultaneous detection of Bordetella bronchiseptica and toxigenic Pasteurella multocida from swine. J. Clin. Microbiol. 36:3342-3346.

Senzilet L.D., Halperin S.A., Spika J.S., Alagaratnam M., Morris A. \& Smith B. 2001. Pertussis is a frequent cause of prolonged cough illness in adults and adolescents. Clin. Infect. Dis. 32:1691-1697.

Stojanov S., Liese J. \& Belohradsky B.H. 2000. Hospitalization and complications in children under 2 years of age with Bordetella pertussis infection. Infection 28:106-110.

Strebel P., Nordin J., Edwards K., Hunt J., Besser J., Burns S., Amundson G., Baughman A. \& Wattigney W. 2001. Population-based incidence of pertussis among adolescents and adults, Minnesota, 1995-1996. J. Infect. Dis. 183:1353-1359.

Underdahl N.R., Socha T.E. \& Doster A.R. 1982. Long-term effect of Bordetella bronchiseptica infection in neonatal pigs. Am. J. Vet. Res. 43:622625 .

Yang W., Pin C., Haibing G., Yang C., Hui L. \& Qigai H. 2009. Loop-mediated isothermal amplification targeting the apxIVA gene for detection of Actinobacillus pleuropneumoniae. FEMS Microbiol. Lett. 300:83-89. 